\title{
TROMBOCYTOSIS FOLLOWING SPLENECTOMY IN PEDIATRIC PATIENTS
}

\author{
Andreea Ligia Dinca ${ }^{1}$, Cristina Oana Marginean ${ }^{1}$, Despina Baghiu ${ }^{1}$, Alina Grama ${ }^{1}$, \\ Horea Gozar ${ }^{2}$, Andreea Durlan', Mihai Stanca ${ }^{3}$, Raluca Damian ${ }^{4}$, \\ Mihaela Chincesan ${ }^{1}$ \\ ${ }^{1}$ Pediatrics Clinic I, University of Medicine and Pharmacy, Targu-Mures \\ ${ }^{2}$ Pediatrics Surgery and Orthopedic Clinic, University of Medicine and Pharmacy, \\ Targu-Mures \\ ${ }^{3}$ University of Medicine and Pharmacy, Targu-Mures \\ ${ }^{4}$ Neonatology Clinic I, Emergency Clinical County Hospital of Targu-Mures
}

\begin{abstract}
Thrombocytosis represents a platelet count over $500.000 / \mathrm{mm}^{3}$.

Objective. The aim of this study is to evaluate the frequency and gravity of reactive thrombocytosis in pediatric patients who underwent splenectomy.

Material and method. We performed a retrospective study including 20 patients (4-16 years old) who underwent splenectomy between 2006-2015. The inclusion criteria in the study were: patients with the age under 18 years, who underwent splenectomy independently by the cause, and who developed afterwards thrombocytosis.

Results. In the studied group $64 \%$ of the splenectomised patients (16) developed a form of thrombocytosis. In 4 cases - severe form (Platelets $>1$ million $/ \mathrm{mm}^{3}$ ) and in 7 cases a mild form. There were not noticed any significant differences regarding the gender repartition of the patients ( 9 were females, and 11 were males). In 13 patients, thrombocytosis disappeared after 30 days, and only in 3 cases, the episode lasted more than 360 days. Thrombotic phenomena were noticed only in one patient from our study group. All the patients benefited from thromboprophylaxis and hydration measures, and 2 cases needed associated treatment with Hydroxyurea.

Conclusions. Our study reveals an increased frequency of thrombocytosis after splenectomy (80\%), with a maximum peak of incidence in 2-10 days following the intervention, thrombocytosis being generally benign and self-limited; still one of the cases experienced thrombotic complications and severe thrombocytosis was more frequent after post-traumatic splenectomy.
\end{abstract}

Keywords: thrombocytosis, splenectomy, child

\section{INTRODUCTION}

Spleen is a complex lymphoid organ that besides the hematopoietic functions, also fulfills other important roles such as the immunologic one, mechanical blood filtration or as cellular reservoir. The indications for splenectomy in pediatrics are precisely defined; the most frequent ones are: hematological diseases, symptomatic splenomegaly, spleen tumors, splenic hemorrhage, neoplasms, or spleen trauma. Postsplenectomy syndrome is characterized by a group of possible complications following the intervention such as: infections, anemia caused by red cell destruction, thrombocytosis (1). Thrombocytosis is defined by a platelet count $>500.000 / \mathrm{mm}^{3}$ and is frequently encountered as an incidental laboratory finding. It can be either pri- mary (clonal) - when it is attributed to a myeloproliferative disorder with abnormal transformation of the hematopoietic cell; it often complicates with thromboembolic phenomena (2). On the other hand, reactive thrombocytosis is secondary to other conditions such as infections, inflammation, trauma, malignancy, or due to splenectomy $(3,4)$. Usually it is a benign and self-limiting disorder which rarely degenerates in massive hemorrhages or thrombotic phenomena (4-6\% of the cases); complications of these type may be due mostly to some qualitative abnormalities of the platelets, and not to quantitative ones $(5,6)$.

The aim of this study is to assess the frequency and the severity of reactive thrombocytosis in pediatric patients who underwent splenectomy.

Corresponding author:

Cristina Oana Marginean, University of Medicine and Pharmacy, 38 Gh. Marinescu Street, Targu-Mures

E-mail: marginean.oana@gmail.com 


\section{MATERIAL AND METHODS}

We performed a retrospective study in which we included splenectomised patients with the age between 4 and 16 years, between 2006-2015, in the Paediatrics Surgery and Orthopaedics Clinic, which were afterwards monitored in the Paediatrics Clinic I, Targu-Mures. From 25 splenectomised patients in these period, only 20 were included in the study according to the inclusion criteria, namely: patients with the age under 18 years, which underwent a splenectomy surgery, independently by the underlying cause (immune thrombocytopenic purpura chronic form, chronic granulocytic leukemia, mycrospherocytic haemolytic anemia, trauma), and which developed thrombocytosis of over 500.000/ $\mathrm{mm}^{3}$ after the surgical intervention. The exclusion criteria consisted in: patients whose parents refused to sign the informed consent or patients with incomplete clinical and biological data.

The aim of the study was the assessment of the patients regarding their clinical and biological status (thrombocytes number, the length between the splenectomy and the appearance of thrombocytosis, the moment of remission, the frequency of the thrombotic episodes), and also regarding the applied therapeutic regimen. The severity of thrombocytosis was classified as it follows: mild form $\left(600-800.000 / \mathrm{mm}^{3}\right)$, medium $(800.000-1 \mathrm{million} /$ $\left.\mathrm{mm}^{3}\right)$ and severe $\left(>1 \mathrm{million} / \mathrm{mm}^{3}\right)$.

\section{RESULTS}

The studied group included 20 patients, aged between 4 and 16 years old. The mean age was 9.6 years old, there was no significant difference between male (11) and female (9) subjects. The underlying condition for which splenectomy was performed was either a hematological one: Chronic Immune Thrombocytopenic Purpura (11 patients),
Microspherocytic Hemolytic Anemia (1 patient), Chronic Granulocytic Leukemia (1 patient); or a traumatic cause -7 patients (Table 1 ).

The splenectomy was scheduled in 13 cases, and was emergently performed in 7 patients. A percentage of $80 \%$ among the patients included in our study (13 subjects) developed a form of thrombocytosis after splenectomy classified as it follows: 7 patients developed a mild form of thrombocytosis, 5 patients a medium form; and 4 patients developed a severe form, presenting over 1 million platelets/ $\mathrm{mm}^{3}$ (Table 2).

The splenectomy due to a hematological cause was more frequently associated with mild forms of thrombocytosis ( 5 out of 9 cases), in comparison with splenectomy of traumatic cause, which associated more frequently severe forms of thrombocytosis ( 3 cases of 7). The medium period from the day of the intervention until the appearance of the high number of platelets was of 3.5 days; the earliest onset was after 2 days, and the latest one after 10 days (Fig. 1).

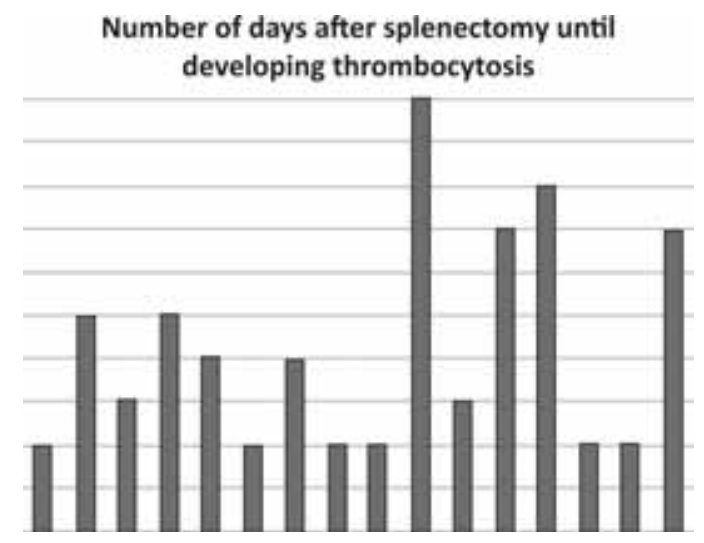

FIGURE 1. The length between splenectomy and thrombocytosis

The episode of thrombocytosis was remitted between 1 and 14 days in 5 of the patients, between

TABLE 1. The underlying condition for which splenectomy was performed

\begin{tabular}{|l|c|c|c|c|}
\hline CAUSE & $\begin{array}{c}\text { Chronic ITP (Immune } \\
\text { Thrombocytopenic Purpura) }\end{array}$ & $\begin{array}{c}\text { Microspherocytic } \\
\text { Hemolytic Anemia }\end{array}$ & $\begin{array}{c}\text { Chronic Granulocytic } \\
\text { Leukemia }\end{array}$ & $\begin{array}{c}\text { Traumatic } \\
\text { cause }\end{array}$ \\
\hline $\begin{array}{l}\text { Patients } \\
\text { number }\end{array}$ & 11 & 1 & 1 & 7 \\
\hline
\end{tabular}

TABLE 2. Platelet count after splenectomy

\begin{tabular}{|l|c|c|c|}
\hline THROMBOCYTOSIS & MILD FORM & MEDIUM FORM & SEVERE FORM \\
\hline PLT count & $600-800.000 / \mathrm{mm}^{3}$ & $800.000-1 \mathrm{milion} / \mathrm{mm}^{3}$ & $>1 \mathrm{milion} / \mathrm{mm}^{3}$ \\
\hline Traumatic cause & 2 & 2 & 3 \\
\hline $\begin{array}{l}\text { Hematological } \\
\text { cause }\end{array}$ & 5 & 3 & 1 \\
\hline Total & $\mathbf{7}$ & $\mathbf{5}$ & $\mathbf{4}$ \\
\hline
\end{tabular}


14 and 30 days in 8 cases and later, after 360 days in 3 of the patients (Fig. 2).

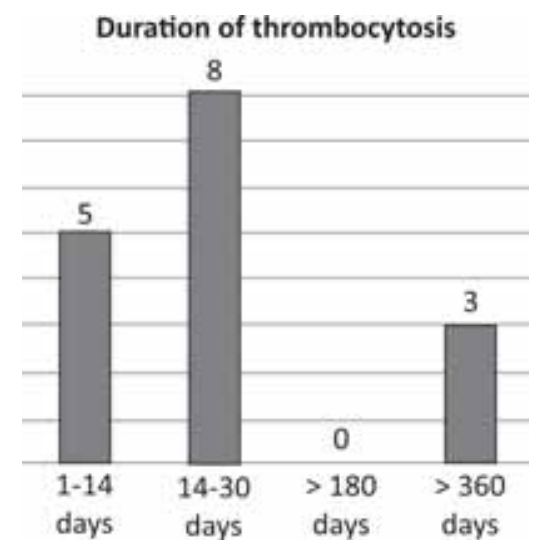

FIGURE 2. The period until the thrombocytosis remission

Thrombotic events were encountered only in one case in who we discovered afterwards also a positive genetic profile for hemophilia.

Regarding the administered treatment, all patients needed auxiliary prophylactic measures or therapy for the decrease of the platelets number. In all cases, we used intense hydration measures and prophylactic therapy with Dipyridamole or Heparin in perfusion/Low molecular weight heparin; in 2 cases we associated cytotoxic therapy Hydreea (Hydroxyurea) in order to reduce the platelet count. We evaluated 10 of our patients after one year, and 4 of them after 2 years after splenectomy.

\section{DISCUSSIONS}

Thrombocytosis is common in childhood, but due to non-specific symptoms, it is rarely diagnosed. Reactive thrombocytosis is the result of an overproduction of thrombopoetic factors (especially Inteleukin-6) that act on megakaryocytes or their precursors; this often happens in infectious, inflammatory, malignant or traumatic processes $(7,8)$. Once the underlying condition is treated, we usually encounter the remission of thrombocytosis, without the risk of other thrombotic events; still, there are cases with platelet hyperaggregation and which need antiaggregant prophylaxis or, in extreme cases, administration of cytotoxic agents (Hydroxyurea, Anagrelid) under careful platelet count monitoring $(9,10)$.

Spleen plays an important role both in regulation and destruction of thrombocytes, this is why thrombocytosis is often associated to hyposplenism (11). The incidence of reactive thrombocytosis after splenectomy was of $80 \%$ in our study group, comparable value to that of $75 \%$ analyzed in a dif- ferent study on 318 patients, existing a difference between the thrombotic events in the cited study $(2.83 \%)$ and the value of $6 \%$ in our group (12). The specialty literature points out an association between the thromboembolic complications and the persistent thrombocytosis after splenectomy in $13 \%$ of the cases (13). The most frequent incriminated risk factors for in favoring the thrombotic complications after splenectomy (thrombocytosis, splenomegaly, thrombophilia) were analyzed in another retrospective study which included 147 patients and pointed out an incidence of 5\% in developing thrombosis of the portal venous system (14). In our study there were recorded thrombotic events in one patient $(6 \%)$, in a 16 year-old teenage girl with severe multiple trauma after a car accident and who needed emergency splenectomy. Afterwards, she developed a severe form of thrombocytosis and it was pointed out a positive genetic profile for thrombophilia. Although there are no guidelines for routine venous thromboprophylaxis in patients who underwent splenectomy and who developed thrombocytosis, antiplatelet drugs are recommended for platelet count $>1.5 \mathrm{million} / \mathrm{mm}^{3}$ when additional risk factors are associated (15). In our study group, 4 of the patients developed severe thrombocytosis, with values of over 1 million platelets $/ \mathrm{mm}^{3}$, and all patients received Dipyridamole as a unique therapy or in association with anticoagulant or cytoreductive agents. For 2 patients, Hydreea (Hydroxyurea) was used, in order to decrease the platelet count. Both in malignant etiologies, that usually associate splenomegaly, and in laparoscopic splenectomies, due to a high risk of venous thrombosis (portal or splenic), studies proved that a low molecular weight heparin anticoagulation for 30 days after surgery represents a benefic prophylaxis for selected patients (16).

Thrombocytosis after splenectomy has a maximum incidence after 1-3 weeks following the intervention, with a slow remission to normal values in weeks, months or even years (8). In the study group, the most frequently, the moment of thrombocytosis was between the $2^{\text {nd }}$ and the $10^{\text {th }}$ day after surgery, with remission in $80 \%$ of the cases in the first 30 days.

\section{CONCLUSIONS}

Our study encountered similar results as in other studies with the same topic, and points out the fact that thrombocytosis after splenectomy is most often benign, and the treatment is an option only in selected case. Reactive thrombocytosis after sple- 
nectomy appeared in $80 \%$ of the patients included in the study, independently of the underlying cause, the severe form of thrombocytosis being more frequent after trauma. Most times (over $87 \%$ of the cases), the episode remitted spontaneously, hydra-

\section{REFERENCES}

1. Connell N.T., Shurin S.B., Schiffman F.J. The spleen and its disorders. In: Hoffman R, Benz E.J Jr., Silberstein L.E et al. Hematology: Basic Principles and Practice. 6th ed. Philadelphia: Elsevier Saunders; 2013: 2252-2265.

2. Syed N., Usman M., Khurshid M. Thrombocytosis: age dependent aetiology and analysis of platelet indices for differential diagnosis. Indian J Pathol and Microbiol. 2007; 50(3): 628-633.

3. Dame C., Sutor A.H. Primary and secondary thrombocytosis in childhood. Br J Haematol. 2005; 129: 165-177.

4. Bleeker J.S., Hogan W.J. Thrombocytosis: diagnostic, evaluation, thrombotic risk stratification, and risk-based management strategies. Thrombosis. 2011; 8: 1-16.

5. Griesshammer M., Bangerter M., Sauer T. et al. Aetiology and clinical significance of thrombocytosis: analysis of 732 patients with an elevated platelet count. J Intern Med. 1999; 245(3): 295-300.

6. Daya S.K., Gowda R.M., Landis W.A. et al. Essential thrombocythemia-related acute ST-segment elevation myocardial infarction. A case report and literature review. Angiology. 2004; 55: 319-23.

7. Santosh-Kumar C.R., Yohannan M.D., Higgy K.E. et al. Trombocytosis in adults: analysis of 777 patients. J. Intern. Med. 1991; 229(6): 493-495.

8. Greer J.P., Foerster J., Rodgers G.M. et al. Wintrobe's Clinical Hematology. Philadelphia: Lippincott Williams \& Wilkins. 2008: 1128-1134. tion in association with antiaggregant and anticoagulant prophylaxis were the only measures imposed. The thrombotic complications were rare (one case only) associating as a risk factor the predisposition for thrombophilia.

9. Harrison C.N., Campbell P.J., Buck G. et al. United Kingdom Medical Research Council Primary Thrombocythemia 1 Study. Hydroxyurea compared with anagrelide in high-risk essential thrombocythemia. N Engl J Med. 2005; 353(1): 33-45.

10. Storen E.C., Tefferi A. Long-term use of anagrelide in young patients with essential thrombocythemia. Blood. 2001; 97(4): 863-866.

11. Bullen A.W., Losowsky M.S. Consequences of impaired splenic function. Clin Sci (Lond). 1979; 57(2): 129-137.

12. Boxer M.A., Braun J., Ellman L. Thromboembolic risk of postsplenectomy thrombocytosis. Arch Surg. 1978; 113 (7): 808-9

13. Cappellini M.D., Grespi E., Cassinerio E. et al. Coagulation and splenectomy: An overview. Ann NY Acad Sci. 2005; 1054: 317-324.

14. Stamou K.M., Toutouzas K.G., Kekis P.B et al. Prospective study of the incidence and risk factors of postsplenectomy thrombosis of the portal, mesenteric, and splenic veins. Arch Surg. $2006 \mathrm{Jul}$; 141(7): 663-669.

15. Linda P. Ha, Mark Arrendondo. Fatal Venous Thromboembolism after Splenectomy: Pathogenesis and Management. J Am Osteopath Assoc. 2012; 112 (5): 291-300

16. Mohren M., Markmann I., Dworschak U. et al. Thromboembolic complications after splenectomy for hematologic diseases. Am J Hematol. 2004; 76 (2):143-147 